\title{
BMJ Open Intrathecal drug delivery systems for the management of chronic non-cancer pain: protocol for a systematic review of economic evaluations
}

\author{
Rui V Duarte, ${ }^{1}$ Tosin Lambe, ${ }^{2}$ Jon H Raphael, ${ }^{3,4}$ Sam Eldabe, ${ }^{5}$ Lazaros Andronis ${ }^{2}$
}

To cite: Duarte RV, Lambe T, Raphael JH, et al. Intrathecal drug delivery systems for the management of chronic non-cancer pain: protocol for a systematic review of economic evaluations. BMJ Open 2016;6:e012285. doi:10.1136/bmjopen-2016012285

- Prepublication history and additional material is available. To view please visit the journal (http://dx.doi.org/ 10.1136/bmjopen-2016012285).

Received 13 April 2016 Revised 15 June 2016 Accepted 27 June 2016

CrossMark

For numbered affiliations see end of article.

Correspondence to

Dr Rui V Duarte;

r.duarte@bham.ac.uk

\section{ABSTRACT}

Introduction: Intrathecal drug delivery (ITDD) systems are an option for the management of patients with chronic non-cancer pain, cancer pain and spasticity. Concerns over their invasiveness and high initial costs have led National Health Service (NHS) England to decommission ITDD for patients with chronic noncancer pain. However, the extent to which this decision is in line with existing economic evidence is unclear. To address this question, we will carry out a systematic review to identify and evaluate the existing evidence on the cost-effectiveness of ITDD for chronic non-cancer pain.

Methods and analysis: A high-sensitivity search strategy will be employed in Cochrane Library, MEDLINE, EMBASE, Web of Science, NHS EED, DARE and HTA. Database searches will be complemented by additional searching techniques. Screening of the results will be performed by 2 reviewers independently using predetermined inclusion and exclusion criteria. Full and partial economic evaluations will be included. Data extraction will be carried out using a form created for the purposes of this review. Quality assessment of all included studies will be performed using recommended checklists.

Ethics and dissemination: Ethical approval is not required as primary data will not be collected. Findings will be disseminated through peer-reviewed publications and conference presentations.

\section{PROSPERO registration number:} CRD42016035266.

\section{INTRODUCTION}

\section{Definition of chronic pain}

Chronic pain is often defined according to the duration of its symptoms, although the time to heal or repair depends on the type of the underlying injury or disease and is extremely variable. For the purposes of consistency, 3 months have been considered an appropriate point to distinguish between acute and chronic non-cancer pain, although 6 months is usually preferred for research

\section{Strengths and limitations of this study}

- First systematic review to investigate the costeffectiveness of intrathecal drug delivery (ITDD) for chronic non-cancer pain.

- The review will consolidate the existing evidence on the cost-effectiveness of ITDD for chronic non-cancer pain.

- It may not be possible to pool estimates of costs and cost-effectiveness.

- Data synthesis will follow good practice recommendations in a narrative summary of health economic studies.

purposes. ${ }^{1}$ Chronic pain can also be characterised as pain that persists past the time point when an injury or disease is healed or repaired has ended. ${ }^{1}$ Healing or repair does not apply to several conditions that are treated as chronic pain, such as rheumatoid arthritis, osteoarthritis or spinal stenosis, and therefore, the concept of chronic pain should be understood as persistent pain that is not responsive to specific treatments or to typical pain relief methods including nonnarcotic analgesics. ${ }^{1}$

\section{Prevalence of chronic pain}

Estimates of the prevalence of chronic pain vary across studies, mainly due to the employed definition of chronic pain and the populations studied. A survey conducted in 15 European countries and Israel defined chronic pain as pain lasting $>6$ months, with pain being experienced several times during the week prior to the survey, and the last pain episode having an intensity of five or more on a 1 (no pain) to 10 (worst pain imaginable) numerical rating scale. ${ }^{2}$ Based on this definition of chronic pain, Breivik et $a l^{2}$ estimated the adult prevalence of chronic pain in the UK to be $13 \%$. The UK Biobank study recruited 503325 people aged 
between 40 and 69 years in England, Scotland and Wales. ${ }^{3}$ No specific characterisation of chronic pain was used in this case; instead, the study relied on individuals reporting their pain as acute or chronic. The study found that the overall prevalence of chronic pain was $42.9 \%$ and increased with age from $39.6 \%$ for people aged between 40 and 44 years to $45.7 \%$ for responders with an age between 65 and 69 years. ${ }^{3}$ The Health Survey for England characterised chronic pain as the experience of pain or discomfort at all of the time or on and off for $>3$ months. ${ }^{4}$ The prevalence of chronic pain was found to be higher in women $(37 \%)$ than in men $(31 \%)$, to increase with age from $14 \%$ of men and $18 \%$ of women aged 16-34 years to $53 \%$ of men and $59 \%$ of women aged 75 years and over, to be higher in those living in the poorest households (40\% of men and $44 \%$ of women) than in the richest households (24\% of men and $30 \%$ of women) and to be higher in those living in more deprived areas (36\% of men and $42 \%$ of women) than in those living in the least deprived areas $(31 \%$ of men and $34 \%$ of women). ${ }^{4}$ The prevalence of chronic pain is higher than that of other common chronic conditions such as diabetes mellitus, which has a considerably lower prevalence of $7 \%$ among men and $4.9 \%$ among women. ${ }^{5}$ Nevertheless, the Chief Medical Officer's Annual Report indicated service provision for pain management to be inadequate and existing services not evenly distributed across the country. ${ }^{6}$

\section{Impact on health and quality of life}

Chronic pain represents a significant health burden that can lead to important reductions in health-related quality of life (QoL). The National Pain Audit 2012 observed that the mean EuroQol index score in people suffering with chronic pain was 0.4 , which is lower than that reported by people with progressive neurological disorders such as Parkinson's disease $(0.432) .{ }^{7}$ It has been reported that, of 291 conditions studied, 4 of the 12 top conditions to cause global disability are persistent pain conditions including neck pain, migraine, arthritis and low back pain. ${ }^{8}$ The latter was found to cause more global disability than any other condition. ${ }^{8}$ Around $65 \%$ of people with chronic pain report difficulty sleeping and $\sim 16 \%$ of them feel that their pain is so bad that sometimes they want to die. ${ }^{6}$

\section{Economic burden of chronic pain}

The economic burden of chronic pain is equally significant. It is estimated that back pain alone costs the UK economy £12.3 billion per year with the cost of pain from all causes being much higher. ${ }^{6}$ In the UK, people with chronic pain report up to five times more general practitioner (GP) appointments than the general population, accounting for 4.6 million GP appointments per year. ${ }^{4}{ }^{9}$ Chronic pain sufferers are seven times more likely to quit their job due to ill health than the general population and chronic pain is the second most common reason to claim incapacity benefit. ${ }^{6}$ Pain was found to have the greatest impact on ability to work as measured by the Brief Pain Inventor; it prevented $40 \%$ of people with chronic pain from working and led to $12 \%$ having to reduce their working hours.

\section{Interventions for chronic pain}

A multitude of strategies are available for the management of chronic non-cancer pain. Treatment strategies for pain start with those with lowest risk and least invasiveness, and progress if the pain is refractory to the treatment selected. ${ }^{10}$ For many patients, it can take several years to identify treatments that can decrease their pain and increase their QoL. Although treatments may initially present some benefits, in the long term, those initial benefits seem to subside. For example, oral opioids, which are frequently used to alleviate postsurgery pain, lead to good short-term pain relief; however, many chronic non-cancer pain patients discontinue long-term therapy due to the need to increase the dose required to obtain similar pain relief, or due to unbearable side-effects. In addition, there is only weak evidence to support the effectiveness of oral opioids in patients who are able to continue opioid therapy in the long term. ${ }^{11}$

Spinal cord stimulation (SCS) and intrathecal drug delivery (ITDD) have been seen as 'last resort' options, which are typically made available to patients who have experienced prolonged periods of pain, sometimes as much as 40 years. ${ }^{12-14}$ The approach to start with those strategies with lowest risk and least invasiveness can be considered as reasonable from a clinical and economic perspective because SCS and ITDD are costly, invasive and carry additional treatment risks. However, it has been observed that chronic pain may cause decrease in brain grey matter, which can be normalised if pain is adequately treated. ${ }^{15}{ }^{16}$ Besides leading to a potential brain grey matter loss, a longer duration of pain is also associated with a decrease in perceived treatment efficacy. ${ }^{13} 17$ Therefore, it is important to identify effective management options for chronic non-cancer pain at an early stage. Expectations may influence a patient's perception of treatment efficacy and it is important for the patients to understand that there is no cure for chronic pain, but there may be options that can help to decrease their pain in a meaningful way, which can lead to improvements in their QoL.

\section{Intrathecal drug delivery}

ITDD has been a recognised treatment for chronic noncancer pain since the first opioid reservoir was implanted in $1981 .{ }^{18}$ Since the administration of opioids is performed directly to a site with opioid receptors, the intrathecal dose required is much lower than that needed when using the oral or epidural route. ${ }^{19}$ The oral and epidural opioid doses are, respectively, $300^{20}$ and $24^{21}$ times higher than the same intrathecal dose. Generally, the direct delivery to the intrathecal space 
and the lower dose required lead to a reduction of sideeffects and enable better analgesia. ${ }^{22}$

An ITDD system consists of a small battery-powered pump implanted into the patients' abdominal area, which delivers medication directly to the intrathecal space through a catheter. The daily dose to be administered is programmed via an external device during refill appointments. The refill procedure and necessary dose adjustments are performed only by doctors or pain nurses with experience in programming the ITDD system.

Candidates for an ITDD undergo a multidisciplinary assessment to evaluate suitability and a trial to assess the patient's response to the intrathecal drug. ITDD trial methodologies are varied and none has proven superior. ${ }^{23-25}$ An ITDD trialling algorithm has been developed suggesting alternative types of trialling strategies based on the type of pain, drug to be administered and time required to evaluate patient's response among other factors. For illustrative purposes, an ITDD trial may be performed using an implanted catheter connected to an external pump, in a single-blind placebocontrolled fashion, with the intrathecal morphine and a saline solution being administered in different days and the patient's response is recorded; patients who report $\geq 50 \%$ pain relief with intrathecal morphine will receive an ITDD implant.

ITDD is used for the management of cancer and noncancer pain and spasticity. ${ }^{26}{ }^{27}$ There are different levels of evidence for the use of ITDD in these different conditions. A randomised controlled trial (RCT) evaluating the effectiveness of ITDD in cancer pain observed improvements in pain control, QoL and significantly less drug toxicity with ITDD when compared with conventional medical management. ${ }^{28}$ Although the current evidence for the use of intrathecal baclofen for the management of spasticity is limited to case series, this treatment is established in the UK. ${ }^{29}$ For chronic non-cancer pain, the evidence on intrathecal delivery of opioids is currently limited to case series and a small RCT suggesting effectiveness of ITDD for pain relief in long-term patients. $^{30}$

National Health Service (NHS) England currently commissions ITDD for the management of cancer pain $^{31}$ and spasticity ${ }^{32}$ but not for the pain of noncancer origin. ${ }^{33}$ Although the limitations of the effectiveness data are undeniable, poor-quality economic evaluations seem to have been used to inform the commissioning decision for chronic non-cancer pain.

\section{Aim and objectives}

The overarching aim of this systematic review is to investigate the cost-effectiveness of ITDD systems using opioids for the management of chronic non-cancer pain.

Towards this aim, this work sets out to

- Search many sources to identify relevant evidence on the cost-effectiveness of ITDD;
- Appraise the quality of the identified studies, and highlight their strengths and limitations;

- Use evidence reported in the studies to determine the cost-effectiveness of this treatment option;

- Discuss the potential policy implications of the findings, especially in relation to future policy reviews of ITDD for chronic non-cancer pain;

- Highlight gaps in the existing evidence, and suggest areas for further research.

\section{METHODS AND ANALYSIS}

This systematic review protocol follows the Preferred Reporting Items for Systematic Reviews and MetaAnalyses for Protocols (PRISMA-P) reporting guidelines. ${ }^{34}$ A PRISMA-P checklist can be found in online supplementary additional file 1 .

\section{Search methods for the identification of studies}

Systematic searches will be conducted to identify relevant economic evaluations of ITDD for the management of chronic non-cancer pain. The searches will be carried out in the following sources:

- Databases: MEDLINE(R) In-Process and Other Non-Indexed Citations and MEDLINE(R) (Ovid), EMBASE (Ovid), Science Citation Index (Web of Science), Conference Proceedings Citation Index (Web of Science), Cochrane Library (Wiley) and the National Health Service (NHS) Centre for Reviews and Dissemination databases NHS EED, DARE and HTA (all via Wiley).

- Grey literature databases: OpenGrey, GreyNet, GreyLit.

Searches in the above bibliographic databases will be complemented by

- Hand searching of reference lists of relevant studies.

- Consultation with experts in the field for additional information on unpublished and ongoing economic evaluations.

Databases will be searched from their inception. Economic studies filters designed by the NHS EED ${ }^{35}$ and $\mathrm{SIGN}^{36}$ to locate economic evaluation studies will be also used. An example of the search strategy to be used in searches in MEDLINE(R) (Ovid) is presented in online supplementary additional file 2. No language restriction will be applied in the searches. Literature search results will be uploaded to and managed using the EndNote V.X7.0.1 software.

\section{Study selection}

The selection criteria described in table 1 will be applied to the citations identified from the literature search. Two reviewers will screen titles and abstracts of all retrieved citations independently. Full texts where compliance with the selection criteria is unclear from title and abstracts will be retrieved. Full papers for studies deemed potentially relevant will be retrieved and selection criteria will be applied. Disagreement will be 
Table 1 Inclusion criteria for the identification of relevant studies

$\begin{array}{ll}\text { Population } & \text { Patients with chronic non-cancer pain lasting for at least } 3 \text { months prior to intervention } \\ \text { Intervention } & \text { ITDD systems using opioids alone or in combination with other agents } \\ \text { Comparator } & \text { Any comparator } \\ \text { Outcomes } & \text { Effectiveness data (ie, patient-reported QoL, pain intensity, disability, patient satisfaction) } \\ & \text { Direct and/or indirect costs to the healthcare system, patients and society } \\ & \text { Items of resource use } \\ \text { Cost per unit of outcome (ie, cost-per-QALY, incremental cost-effectiveness ratio) }\end{array}$

ITDD, intrathecal drug delivery; QALY, quality-adjusted life years; QoL, quality of life.

resolved by discussion and consensus between the two reviewers. A third reviewer will be involved if dissenting opinions are observed and consensus is not reached. The study selection process will be summarised in a PRISMA flow diagram.

\section{Data extraction}

Two reviewers will extract relevant information independently using an extraction form developed specifically for the purposes of this study. A third reviewer will assess the extracted data to ensure accuracy. Disagreements will be resolved by discussion. Information will be extracted in relation to the following factors: (1) general information including study ID, author, year, funding source, country, setting and study design; (2) recruitment details, sample size, demographic characteristics (age, gender) and baseline health data (diagnosis, comorbidities); (3) interventions, effectiveness data and cost data; (4) type of economic evaluation, perspective, time horizon and measure of benefit; (5) quality assessment; (6) results; (7) analysis of uncertainty and (8) conclusions. The outcomes for which data will be sought were selected taking into account the data necessary to conduct an economic evaluation.

\section{Quality assessment}

Quality assessment of all included studies will be performed using the Evers checklist (all economic evaluations) $)^{38}$ and the Philips checklist for model-based economic studies ${ }^{39}$ as recommended in the Cochrane Handbook for Systematic Reviews of Interventions. ${ }^{40}$ Two reviewers will independently assess the quality of the included studies. Any discrepancies will be resolved by discussion and consensus between the two reviewers and if necessary consultation of a third reviewer. Quality assessment of included studies will be presented in a table format.

\section{Data synthesis and reporting}

The results section will be organised taking into consideration the good practice recommendations in a narrative summary of health economic studies from the Cochrane Handbook for Systematic Reviews. ${ }^{41}$ The recommendations advise detailed reports of methods such as the study design; time horizon adopted; currency and price year; measures of benefits used; assessment of uncertainty; methodological quality and limitations of all studies included in the review. The actual results from the studies will be reported in a separate results subsection.

To facilitate the comparison of estimates reported in different studies, monetary values reported in all the identified studies will be converted to UK pounds sterling $(£)$ at 2015 price year. Conversion of cost estimates will be performed using the CCEMG-EPPI-Centre Cost Converter web-based tool V.1.4. The CCEMGEPPI-Centre Cost Converter tool takes into consideration international exchange rates based on Purchasing Power Parities and gross domestic product deflator values as recommended in the economics evidence section of the Cochrane Handbook for Systematic Reviews. ${ }^{41}$

\section{ETHICS AND DISSEMINATION}

Ethical approval is not required as primary data will not be collected. Findings will be disseminated through peerreviewed publications and conference presentations.

\section{DISCUSSION}

Alongside clinical evidence, economic evaluations are an important tool for policymakers. Taking into consideration budget limitations, it is imperative that decisions to recommend a treatment and make it available to NHS patients are underpinned by robust evidence on its clinical effectiveness and cost-effectiveness. In some occasions, it is possible that important evidence with the potential to influence decisions may not have been identified. Therefore, if there is uncertainty about the clinical effectiveness or cost-effectiveness of a treatment, a systematic review should be commissioned prior to a decision. Recently, NHS England has decommissioned the use of ITDD for the management of chronic non-cancer pain. ${ }^{33}$ Poor-quality economic evaluations seem to have been used to inform the commissioning decision. The authors do not state that this review alone or the use of better quality economic evaluations would lead to a change in the decision, due to the limited evidence for effectiveness. However, with better effectiveness data, the 
assessment of poor economic evaluations (when better evidence is available) could tip the decision towards noncommissioning and as a consequence, access to patients with non-cancer pain that could potentially benefit from ITDD would be denied.

\section{Author affiliations}

${ }^{1}$ Department of Public Health, Epidemiology and Biostatistics, Institute of Applied Health Research, University of Birmingham, Birmingham, UK

${ }^{2}$ Health Economics Unit, Institute of Applied Health Research, University of Birmingham, Birmingham, UK

${ }^{3}$ Faculty of Health, Birmingham City University, Birmingham, UK

${ }^{4}$ Department of Pain Management, Russells Hall Hospital, Dudley, UK

${ }^{5}$ Department of Pain and Anaesthesia, The James Cook University Hospital,

Middlesbrough, UK

Contributors RVD wrote the protocol and conceived the initial idea for the study. TL, JHR, SE and LA critically appraised the protocol and contributed to its development. All authors approved the final version and took responsibility for its content.

Funding RVD has received funding from the Neuromodulation Society of the United Kingdom and Ireland (NSUKI) in support of this study. The funder had no role in study design, data collection and analysis, decision to publish or preparation of the manuscript.

Disclaimer The views expressed here are those of the authors and not necessarily those of NSUKI.

Competing interests None declared.

Provenance and peer review Not commissioned; externally peer reviewed.

Open Access This is an Open Access article distributed in accordance with the Creative Commons Attribution Non Commercial (CC BY-NC 4.0) license, which permits others to distribute, remix, adapt, build upon this work noncommercially, and license their derivative works on different terms, provided the original work is properly cited and the use is non-commercial. See: http:// creativecommons.org/licenses/by-nc/4.0/

\section{REFERENCES}

1. Loeser JD, Arendt-Nielsen L, Baron R, et al. Classification of chronic pain: descriptions of chronic pain syndromes and definitions of pain terms / prepared by the International Association for the Study of Pain Task Force on Taxonomy. 2nd rev edn. Seattle: IASP Press, 2012.

2. Breivik $\mathrm{H}$, Collett $B$, Ventafridda $\mathrm{V}$, et al. Survey of chronic pain in Europe: prevalence, impact on daily life, and treatment. Eur J Pain 2006; $10: 287-333$

3. Macfarlane GJ, Beasley M, Smith BH, et al. Can large surveys conducted on highly selected populations provide valid information on the epidemiology of common health conditions? An analysis of UK Biobank data on musculoskeletal pain. Br J Pain 2015;9:203-12.

4. Bridges S. Chapter 9: chronic pain. In: Craig R, Mindell J, eds. Health survey for England 2011, health, social care and lifestyles. London: The Health and Social Care Information Centre, 2012:1-33.

5. Moody A. Chapter 4: diabetes and hyperglycaemia. In: Craig R, Mindell J, eds. Health survey for England 2011, health, social care and lifestyles. London: The Health and Social Care Information Centre, 2012:1-28.

6. Donaldson L. Pain: breaking through the barrier. In: Donaldson L, ed. 150 years of the annual report of the chief medical officer: on the state of public health 2008. London: Department of Health, 2009:32-9.

7. Price C, Hoggart B, Olukoga O, et al. National Pain Audit Final Report 2012-2012. London: Healthcare Quality Improvement Partnership, British Pain Society and Dr Foster Intelligence, 2012.

8. Hoy D, March L, Brooks P, et al. The global burden of low back pain: estimates from the Global Burden of Disease 2010 study. Ann Rheum Dis 2014;73:968-74.

9. Phillips CJ. The cost and burden of chronic pain. Rev Pain 2009;3:2-5.

10. Krames ES, Olson K. Clinical realities and economic considerations: patient selection in intrathecal therapy. J Pain Symptom Manage 1997; $14:$ S3-13.
11. Noble M, Treadwell JR, Tregear SJ, et al. Long-term opioid management for chronic noncancer pain. Cochrane Database Syst Rev 2010:CD006605.

12. Duarte RV, Raphael JH, Sparkes E, et al. Long-term intrathecal drug administration for chronic nonmalignant pain. $J$ Neurosurg Anesthesiol 2012;24:63-70.

13. Sparkes E, Duarte RV, Mann S, et al. Analysis of psychological characteristics impacting spinal cord stimulation treatment outcomes: a prospective assessment. Pain Physician 2015;18:E369-77.

14. Mann SA, Sparkes E, Duarte RV, et al. Attrition with spinal cord stimulation. Br J Neurosurg 2015;29:823-8.

15. May A. Chronic pain may change the structure of the brain. Pain 2008:137:7-15.

16. Rodriguez-Raecke $\mathrm{R}$, Niemeier $\mathrm{A}$, Ihle $\mathrm{K}$, et al. Brain gray matter decrease in chronic pain is the consequence and not the cause of pain. J Neurosci 2009;29:13746-50.

17. Kumar K, Hunter G, Demeria D. Spinal cord stimulation in treatment of chronic benign pain: challenges in treatment planning and present status, a 22-year experience. Neurosurgery 2006;58:481-96.

18. Onofrio BM, Yaksh TL, Arnold PG. Continuous low-dose intrathecal morphine administration in the treatment of chronic pain of malignant origin. Mayo Clin Proc 1981;56:516-20.

19. Coombs DW, Saunders RL, Gaylor MS, et al. Relief of continuous chronic pain by intraspinal narcotics infusion via an implanted reservoir. JAMA 1983;250:2336-9.

20. Lamer TJ. Treatment of cancer-related pain: when orally administered medications fail. Mayo Clin Proc 1994;69:473-80.

21. Nordberg G, Hedner T, Mellstrand T, et al. Pharmacokinetic aspects of intrathecal morphine analgesia. Anesthesiology 1984;60:448-54.

22. Prager JP. Neuraxial medication delivery: the development and maturity of a concept for treating chronic pain of spinal origin. Spine 2002;27:2593-605.

23. Deer T, Winkelmüller W, Erdine S, et al. Intrathecal therapy for cancer and nonmalignant pain: patient selection and patient management. Neuromodulation 1999;2:55-66.

24. Deer TR, Prager J, Levy R, et al. Polyanalgesic Consensus Conference-2012: recommendations on trialing for intrathecal (intraspinal) drug delivery: report of an interdisciplinary expert panel. Neuromodulation 2012;15:420-35; discussion 435.

25. Deer TR, Hayek S, Pope J, et al. The Polyanalgesic Consensus Conference (PACC) guidelines for intrathecal drug delivery infusion system trialing. Neuromodulation 2016. In press.

26. Duarte R, Raphael J, Eldabe S. Intrathecal drug delivery for the management of pain and spasticity in adults: an executive summary of the British Pain Society's recommendations for best clinical practice. Br J Pain 2016;10:67-9.

27. Eldabe S, Duarte R, Raphael J, et al. Intrathecal drug delivery for the management of pain and spasticity in adults; recommendations for best clinical practice. London: British Pain Society, 2015.

28. Smith TJ, Staats PS, Deer T, et al. Implantable Drug Delivery Systems Study Group. Randomized clinical trial of an implantable drug delivery system compared with comprehensive medical management for refractory cancer pain: impact on pain, drug-related toxicity, and survival. J Clin Oncol 2002;20:4040-9.

29. Narendran RC, Duarte RV, Valyi A, et al. The need for and provision of intrathecal baclofen therapy for the management of spasticity in England: an assessment of the Hospital Episode Statistics database. BMJ Open 2015;5:e007517.

30. Raphael JH, Duarte RV, Southall JL, et al. Randomised, double-blind controlled trial by dose reduction of implanted intrathecal morphine delivery in chronic non-cancer pain. BMJ Open 2013;3:e003061.

31. NHS England. Clinical commissioning policy: intrathecal pumps for treatment of severe cancer pain. 2015. https://www.england.nhs.uk/ commissioning/wp-content/uploads/sites/12/2015/10/ d08pb-intra-pumps-trtmnt.pdf (accessed 10 Dec 2015).

32. NHS Commissioning Board. Clinical commissioning policy: intrathecal baclofen (ITB). 2013. https://www.england.nhs.uk/ wp-content/uploads/2013/04/d04-p-c.pdf (accessed 10 Dec 2015).

33. NHS England. Clinical commissioning policy: intrathecal pumps for treatment of severe chronic pain. 2015. https://www.england.nhs.uk commissioning/wp-content/uploads/sites/12/2015/10/ d08pa-intrathecal-pumps-oct15.pdf (accessed 10 Dec 2015).

34. Shamseer L, Moher D, Clarke M, et al., PRISMA-P Group. Preferred reporting items for systematic review and meta-analysis protocols (PRISMA-P) 2015: elaboration and explanation. BMJ 2015;349: g7647.

35. Centre for Reviews and Dissemination. Search strategies. 2015. http://www.crd.york.ac.uk/crdweb/searchstrategies.asp (accessed 17 Dec 2015). 
36. Scottish Intercollegiate Guidelines Network. Search filters. 2015. http://www.sign.ac.uk/methodology/filters.html (accessed $17 \mathrm{Dec}$ 2015).

37. Drummond MF, Sculpher MJ, Torrance GW. Methods for the economic evaluation of health care programmes. 3rd edn. Oxford: Oxford University Press, 2005.

38. Evers $\mathrm{S}$, Goossens $\mathrm{M}$, de Vet $\mathrm{H}$, et al. Criteria list for assessment of methodological quality of economic evaluations: consensus on health economic criteria. Int J Technol Assess Health Care 2005;21:240-5.

39. Philips Z, Ginnelly L, Sculpher M, et al. Review of guidelines for good practice in decision-analytic modelling in health technology assessment. Health Technol Assess 2004;8:iii-iv, ix-xi, $1-158$.

40. Higgins J, Green S. Cochrane handbook for systematic reviews of interventions. Version 5.1.0. [updated March 2011]. Chichester: John Wiley \& Sons, 2011

41. Shemilt I, Mugford M, Byford S, et al., on behalf of the Campbell and Cochrane Economics Methods Group. Chapter 15:

incorporating economics evidence. In: Higgins JPT, Green S, eds. Cochrane handbook for systematic reviews of interventions. Version 5.1.0 [updated March 2011]. Chichester: John Wiley \& Sons, 2011. http://handbook.cochrane.org/chapter_15/ 15 10 chapter information.htm 